\title{
Helical magnetic effect and the chiral anomaly
}

\author{
Naoki Yamamoto $\odot^{1}$ and Di-Lun Yang ${ }^{1,2}$ \\ ${ }^{1}$ Department of Physics, Keio University, Yokohama 223-8522, Japan \\ ${ }^{2}$ Institute of Physics, Academia Sinica, Taipei 11529, Taiwan
}

(Received 4 April 2021; accepted 6 May 2021; published 4 June 2021)

\begin{abstract}
In the presence of the fluid helicity $\boldsymbol{v} \cdot \boldsymbol{\omega}$, the magnetic field induces an electric current of the form $\boldsymbol{j}=C_{\mathrm{HME}}(\boldsymbol{v} \cdot \boldsymbol{\omega}) \boldsymbol{B}$. This is the helical magnetic effect (HME). We show that for massless Dirac fermions with charge $e=1$, the transport coefficient $C_{\mathrm{HME}}$ is fixed by the chiral anomaly coefficient $C=1 /\left(2 \pi^{2}\right)$ as $C_{\mathrm{HME}}=C / 2$ independently of interactions. We show the conjecture that the coefficient of the magnetovorticity coupling for the local vector charge, $n=C_{B \omega} \boldsymbol{B} \cdot \boldsymbol{\omega}$, is related to the chiral anomaly coefficient as $C_{B \omega}=C / 2$. We also discuss the condition for the emergence of the helical plasma instability that originates from the HME.
\end{abstract}

DOI: 10.1103/PhysRevD.103.125003

\section{INTRODUCTION}

Chiral transport phenomena in relativistic chiral matter have attracted growing interests in various physical systems, such as heavy ion collisions [1], early Universe [2], core-collapse supernovae [3], and Weyl semimetals [4]. One prototype example is the chiral magnetic effect (CME) [5-8] - the electric current along a magnetic field in the presence of a chirality imbalance of fermions, characterized by a chiral chemical potential $\mu_{5}$.

In Ref. [3], it was pointed out that in the presence of a finite helicity, such as fluid helicity $\boldsymbol{v} \cdot \boldsymbol{\omega},{ }^{1}$ an electric current is induced by the magnetic field $\boldsymbol{B}$ even without $\mu_{5}$ :

$$
\boldsymbol{j}=C_{\mathrm{HME}}(\boldsymbol{v} \cdot \boldsymbol{\omega}) \boldsymbol{B},
$$

where $\boldsymbol{v}$ is the local fluid velocity and $\boldsymbol{\omega}=\frac{1}{2} \boldsymbol{\nabla} \times \boldsymbol{v}$ is the vorticity. This was coined the helical magnetic effect (HME). ${ }^{2}$ However, the transport coefficient $C_{\mathrm{HME}}$ has not been determined so far, largely because this is a nonlinear nonequilibrium transport in terms of external fields $\boldsymbol{v}, \boldsymbol{\omega}$, and $\boldsymbol{B}$.

In this paper, we show that the transport coefficient $C_{\mathrm{HME}}$ for massless Dirac fermions is fixed by the coefficient of the chiral anomaly $C$ as

\footnotetext{
${ }^{1}$ The fluid helicity has been known to have an important role in the hydrodynamics [9], and in particular, in the turbulence [10].

${ }^{2}$ For the HME and helical vortical effects in other contexts, see Refs. [11,12].

Published by the American Physical Society under the terms of the Creative Commons Attribution 4.0 International license. Further distribution of this work must maintain attribution to the author(s) and the published article's title, journal citation, and DOI. Funded by SCOAP.
}

$$
C_{\mathrm{HME}}=\frac{C}{2}, \quad C \equiv \frac{1}{2 \pi^{2}} .
$$

Along the way, we also show the conjecture in Ref. [13] that the coefficient of the magnetovorticity coupling for the local vector charge,

$$
n=C_{B \omega} \boldsymbol{B} \cdot \omega,
$$

is connected to the chiral anomaly coefficient $C$ as

$$
C_{B \omega}=\frac{C}{2} .
$$

While $C_{B \omega}=1 /\left(4 \pi^{2}\right)$ was derived for noninteracting massless Dirac fermions in the homogeneous magnetic field in Ref. [13], our derivation shows that Eq. (4) is exact even in the presence of interactions for generic inhomogeneous magnetic fields.

Our derivation is based on the idea that, under the assumption that the system of interest is in local thermal equilibrium, the vorticity is introduced as a fictitious spacetime torsion [14,15], which, in turn can be regarded as a background axial gauge field [16]. This is in spirit similar to Luttinger's argument that a temperature gradient can be introduced as a fictitious gravitational field [17]. In this way, we will show that the HME may be understood as a kind of the chiral torsional effect recently discussed in literature [15,18-20].

In Ref. [3], it was also argued that the presence of the HME leads to a new type of plasma instability, coined the helical plasma instability (HPI), in the same way that the CME induces the chiral plasma instability (CPI) [21]. In this paper, we also discuss under which conditions the HPI can appear. 
This paper is organized as follows. In Sec. II, we review the Chern-Simons currents for massless Dirac fermions. In Sec. III, we argue that the vorticity can be realized as an axial gauge field. In Sec. IV, we show that the coefficients of the HMI and other related vorticity-induced effects are fixed by the anomaly coefficient. In Sec. V, we discuss the condition for the emergence of the HPI. In Sec. VI, we make concluding remarks and discuss open questions.

Throughout the paper, we use the natural units $\hbar=c=k_{\mathrm{B}}=1$. We absorb the elementary charge $e$ into the definition of the gauge field $A_{\mu}$ unless stated otherwise. We use the Minkowski metric $\eta_{\mu \nu}=(+1,-1,-1,-1)$ and define the totally antisymmetric tensor $\epsilon^{\mu \nu \alpha \beta}$ such that $\epsilon^{0123}=+1$. We also introduce the notations $A_{[\mu} B_{\nu]} \equiv$ $A_{\mu} B_{\nu}-A_{\nu} B_{\mu}$ and $A_{(\mu} B_{\nu)} \equiv A_{\mu} B_{\nu}+A_{\nu} B_{\mu}$.

\section{CHERN-SIMONS CURRENTS}

We first review the Chern-Simons currents for massless Dirac fermions that will be used in the following discussions; see, e.g., Ref. [22] for a recent review. We consider a system of massless Dirac fermions coupled to the vector and axial gauge fields, $A^{\mu}$ and $A_{5}^{\mu}$ :

$$
\mathcal{L}=\bar{\psi}\left(\mathrm{i} \gamma^{\mu} \partial_{\mu}-\gamma^{\mu} A_{\mu}-\gamma^{\mu} \gamma^{5} A_{\mu}^{5}\right) \psi .
$$

One may alternatively regard this theory as right- and lefthanded fermions coupled to right- and left-handed gauge fields, $A_{\mathrm{R}}^{\mu} \equiv A^{\mu}+A_{5}^{\mu}$ and $A_{\mathrm{L}}^{\mu} \equiv A^{\mu}-A_{5}^{\mu}$, respectively,

$$
\mathcal{L}=\psi_{\mathrm{R}}^{\dagger} \mathrm{i} \sigma^{\mu}\left(\partial_{\mu}+\mathrm{i} A_{\mu}^{\mathrm{R}}\right) \psi_{\mathrm{R}}+\psi_{\mathrm{L}}^{\dagger} \mathrm{i} \bar{\sigma}^{\mu}\left(\partial_{\mu}+\mathrm{i} A_{\mu}^{\mathrm{L}}\right) \psi_{\mathrm{L}},
$$

where $\psi_{\mathrm{R}, \mathrm{L}}$ are right- and left-handed fermions, $\sigma^{\mu}=(1, \boldsymbol{\sigma})$, and $\bar{\sigma}^{\mu}=(1,-\boldsymbol{\sigma})$ with $\sigma^{i}(i=1,2,3)$ being the Pauli matrices. One can then derive the covariant anomalies for the right- and left-handed sector as

$$
\partial_{\mu} j_{\chi}^{\mu}=\mp \frac{C}{16} \epsilon^{\mu \nu \alpha \beta} F_{\mu \nu}^{\chi} F_{\alpha \beta}^{\chi} \quad(\chi=\mathrm{R}, \mathrm{L}),
$$

where $F_{\mu \nu}^{\chi}=\partial_{\mu} A_{\nu}^{\chi}-\partial_{\nu} A_{\mu}^{\chi}$. By adding and subtracting the right- and left-handed sectors, we have

$$
\begin{gathered}
\partial_{\mu} j^{\mu}=-\frac{C}{4} \epsilon^{\mu \nu \alpha \beta} F_{\mu \nu} F_{\alpha \beta}^{5}, \\
\partial_{\mu} j_{5}^{\mu}=-\frac{C}{8} \epsilon^{\mu \nu \alpha \beta}\left(F_{\mu \nu} F_{\alpha \beta}+F_{\mu \nu}^{5} F_{\alpha \beta}^{5}\right),
\end{gathered}
$$

where $F_{\mu \nu}=\partial_{\mu} A_{\nu}-\partial_{\nu} A_{\mu}$ and $F_{\mu \nu}^{5}=\partial_{\mu} A_{\nu}^{5}-\partial_{\nu} A_{\mu}^{5}$.

We will be interested in the case where $A_{\mu}$ is dynamical while $A_{\mu}^{5}$ is external. In this case, Eq. (8) is problematic in the sense that it is inconsistent with the gauge symmetry. In fact, the equation of motion for $A_{\mu}$ is given by Maxwell's equations

$$
\partial_{\nu} F^{\nu \mu}=j^{\mu},
$$

and so

$$
\partial_{\mu} j^{\mu}=\partial_{\mu} \partial_{\nu} F^{\nu \mu}=0 .
$$

One then finds that Eq. (8) is inconsistent with Eq. (11).

The way out is well known. We can add a topological current called the Chern-Simons current

$$
j_{\mathrm{CS}}^{\mu}=\frac{C}{2} \epsilon^{\mu \nu \alpha \beta} A_{\nu}^{5} F_{\alpha \beta}
$$

into $j^{\mu}$ such that the right-hand side of Eq. (8) is cancelled out (namely, $\partial_{\mu} \tilde{j}^{\mu}=0$, where $\tilde{j}^{\mu}=j^{\mu}+j_{\mathrm{CS}}^{\mu}$ ). The temporal and spatial components read

$$
\begin{aligned}
& n_{\mathrm{CS}}=C A^{5} \cdot \boldsymbol{B}, \\
& \boldsymbol{j}_{\mathrm{CS}}=C A_{0}^{5} \boldsymbol{B}+C \boldsymbol{E} \times \boldsymbol{A}_{5},
\end{aligned}
$$

respectively. Note that all the coefficients appearing in Eqs. (13) and (14) are fixed by the anomaly coefficient $C$ by construction.

One might wonder what the physical realizations of the axial gauge field $A_{\mu}^{5}$ are. There are in fact systems where $A_{\mu}^{5}$ appears emergently. For example, in Weyl semimetals, $A_{0}^{5}=b_{0}$ and $A^{5}=\boldsymbol{b}$, where $b_{0}$ and $\boldsymbol{b}$ correspond to the energy and momentum separations between two Weyl nodes. As a result, the electric current at finite $\mu_{5}$ is given by

$$
\boldsymbol{j}=C\left(\mu_{5}+b_{0}\right) \boldsymbol{B}+C \boldsymbol{E} \times \boldsymbol{b},
$$

where the first term is the CME $[23,24]$ and the second is the anomalous Hall effect [25-28]. ${ }^{3}$ In particular, in equilibrium where $\mu_{5}=-b_{0}$, the CME vanishes as is consistent with the generalized Bloch theorem [31].

We will next argue that the vorticity can also be understood as an emergent axial gauge field.

\section{VORTICITY AS AN AXIAL GAUGE FIELD}

We are interested in the hydrodynamic regime of a gauge theory with finite vorticity $\boldsymbol{\omega} \neq \mathbf{0}$. Below we will use the following two facts: (i) the vorticity is introduced as a fictitious spacetime torsion $[14,15]$, and (ii) the torsion can be regarded as an axial gauge field [16]. As a result, the vorticity can be realized as an axial gauge field.

To see the fact (ii) first, consider a spacetime with torsion defined by $T_{\mu \nu}^{\rho} \equiv \tilde{\Gamma}_{[\mu \nu]}^{\rho}$, where $\tilde{\Gamma}_{\mu \nu}^{\rho}$ is a nonsymmetric affine connection satisfying $\tilde{\Gamma}_{\mu \nu}^{\rho}=\Gamma_{\mu \nu}^{\rho}+K_{\mu \nu}^{\rho}$, with $\Gamma_{\mu \nu}^{\rho}$ being the symmetric Christoffel symbol and $K_{\mu \nu \rho} \equiv \frac{1}{2}\left(T_{\mu \nu \rho}-T_{\nu \mu \rho}-\right.$ $\left.T_{\rho \mu \nu}\right)$ the contorsion tensor. The action for massless Dirac fermions is [16]

\footnotetext{
${ }^{3}$ In the context of Weyl semimetals, the importance of the Chern-Simons contributions is stressed in Refs. [29,30].
} 


$$
S=\int \mathrm{d}^{4} x \sqrt{-g} \frac{\mathrm{i}}{2}\left(\bar{\psi} \gamma^{\mu} \tilde{\nabla}_{\mu} \psi-\tilde{\nabla}_{\mu} \bar{\psi} \gamma^{\mu} \psi\right)
$$

where $g$ is the determinant of the spacetime metric and

$$
\begin{aligned}
& \tilde{\nabla}_{\mu} \psi=\partial_{\mu} \psi+\frac{\mathrm{i}}{4} \tilde{\omega}_{\mu}^{\hat{a} \hat{b}} \sigma_{\hat{a} \hat{b}} \psi, \\
& \tilde{\nabla}_{\mu} \bar{\psi}=\partial_{\mu} \bar{\psi}-\frac{\mathrm{i}}{4} \bar{\psi} \tilde{\omega}_{\mu}^{\hat{a} \hat{b}} \sigma_{\hat{a} \hat{b}}, \\
& \tilde{\omega}_{\mu \hat{a} \hat{b}}=\omega_{\mu \hat{a} \hat{b}}+K_{\cdot \lambda \mu}^{\alpha} e_{\hat{a}}^{\lambda} e_{\hat{b} \alpha} .
\end{aligned}
$$

Here, $\omega_{\mu \hat{a} \hat{b}}=e_{\hat{b} \alpha} \partial_{\mu} e_{\hat{a}}^{\alpha}+\Gamma_{\lambda \mu}^{\alpha} e_{\hat{a}}^{\lambda} e_{\hat{b} \alpha}$ is the spinor connection in the spacetime without torsion, $\sigma_{\hat{a} \hat{b}}=\frac{i}{2}\left[\gamma_{\hat{a}}, \gamma_{\hat{b}}\right]$ with $\gamma^{\hat{a}}$ being the usual $\gamma$ matrix in flat spacetime, and $\gamma^{\mu}=e_{\hat{a}}^{\mu} \gamma^{\hat{a}}$ with $e_{\hat{a}}^{\mu}$ the vierbein satisfying $e_{\mu}^{\hat{a}} e^{\hat{b} \mu}=\eta^{\hat{a} \hat{b}}$ and $e_{\mu}^{\hat{a}} e_{\nu}^{\hat{a}}=g_{\mu \nu}$. In the following, we will be interested in the torsional effects in flat spacetime. In this case, the Lagrangian can be rewritten as [16]

$$
\mathcal{L}=\bar{\psi}\left(\mathrm{i} \gamma^{\mu} \partial_{\mu}-\frac{1}{8} \gamma^{\mu} \gamma^{5} S_{\mu}\right) \psi
$$

where $S_{\nu} \equiv \epsilon_{\alpha \beta \mu \nu} T^{\alpha \beta \mu}$.

To see the fact (i) above, we consider the metric

$$
\mathrm{d} s^{2}=\mathrm{d} t^{2}+2 v \cdot \mathrm{d} x \mathrm{~d} t-\mathrm{d} x^{2}
$$

up to $O\left(\boldsymbol{v}^{1}\right)$. We here take $\boldsymbol{v}$ to depend on the coordinate $\boldsymbol{x}$ (but not on time $t$ ), $\boldsymbol{v}=\boldsymbol{v}(\boldsymbol{x})$, and in the following, we will focus on the terms related to the vorticity $\boldsymbol{\omega}=\frac{1}{2} \boldsymbol{\nabla} \times \boldsymbol{v} \neq \mathbf{0}$ in the local rest frame of the fluid. ${ }^{4}$ The vierbein corresponding to the metric (20) is given by [15]

$e_{0}^{\hat{0}}=1, \quad e_{i}^{\hat{0}}=-v_{i}, \quad e_{0}^{\hat{i}}=0, \quad e_{j}^{\hat{i}}=\delta_{j}^{\hat{i}}$.

We now impose vierbein postulate $\tilde{\nabla}_{\mu} e_{\nu}^{\hat{a}}=0$ for $\omega_{\mu \hat{a} \hat{b}}=0$. From the antisymmetric and symmetric parts of this postulate with respect to the indices $\mu$ and $\nu$, we obtain

$$
\begin{aligned}
T_{\mu \nu}^{\hat{a}} & =\partial_{[\mu} e_{\nu]}^{\hat{a}}, \\
\partial_{(\mu} e_{\nu)}^{\hat{a}} & =2 \Gamma_{\mu \nu}^{\lambda} e_{\lambda}^{\hat{a}}-T_{(\mu \lambda \nu)} e^{\hat{a} \lambda}
\end{aligned}
$$

where $T_{\mu \nu}^{\hat{a}}=T_{\mu \nu}^{\rho} e_{\rho}^{\hat{a}}$. For the specific choice of the vierbein in Eq. (21), we have

$$
T_{i j}^{\hat{0}}=-\partial_{[i} v_{j]}=-2 \epsilon_{i j k} \omega^{k},
$$

where $\epsilon_{i j k} \equiv \epsilon_{0 i j k}$ and the other components of $T_{\mu \nu}^{\hat{a}}$ vanish. One can also check that the $(\hat{a}, \mu, \nu)=(\hat{0}, 0, i),(\hat{0}, i, 0)$

\footnotetext{
${ }^{4}$ The same setup is considered to derive the transport coefficient of the chiral vortical effect (CVE) in Ref. [32].
}

components of Eq. (22) lead to the constraint $\partial_{t} \boldsymbol{v}=\mathbf{0}$ while Eq. (22) is automatically satisfied for the other components at the order of $O\left(\boldsymbol{v}^{1}\right)$. Consequently, we have

$$
S_{\mu}=\left(0,4 \omega_{i}\right) .
$$

So far, we have considered the local rest frame of the fluid. The expression of $S^{\mu}$ in the generic inertial frame, where the local fluid four velocity is $u^{\mu}=\gamma(1, v)$ with $\gamma=\left(1-v^{2}\right)^{-1 / 2}$, can be obtained by performing a Lorentz boost as

$$
S_{\mu}=4 \omega_{\mu},
$$

where $\omega_{\mu}=\frac{1}{2} \epsilon_{\mu \nu \alpha \beta} u^{\nu} \partial^{\alpha} u^{\beta}$.

In summary, we see that the vorticity can be introduced as an emergent background axial gauge field

$$
A_{\mu}^{5} \equiv \frac{1}{8} S_{\mu}=\frac{1}{2} \omega_{\mu}
$$

in the generic inertial frame.

\section{HELICAL MAGNETIC EFFECT AND OTHER VORTICITY-INDUCED EFFECTS}

We are now ready to derive the vorticity-induced effects in the hydrodynamic regime of a gauge theory by combining the results in the previous sections.

By inserting Eq. (27) into Eq. (12), we find

$$
j_{\mathrm{CS}}^{\mu}=\frac{C}{4} \epsilon^{\mu \nu \alpha \beta} \omega_{\nu} F_{\alpha \beta} .
$$

We can also rewrite Eq. (28) in terms of the electromagnetic fields defined in the fluid rest frame, $E^{\mu}=F^{\mu \nu} u_{\nu}$ and $B^{\mu}=\frac{1}{2} \epsilon^{\mu \nu \alpha \beta} u_{\nu} F^{\alpha \beta}$. By using the decomposition

$$
F_{\mu \nu}=E_{(\mu} u_{\nu)}+\epsilon_{\mu \nu \alpha \beta} u^{\alpha} B^{\beta}
$$

and the identity $u \cdot \omega=0$, we obtain

$$
j_{\mathrm{CS}}^{\mu}=-\frac{C}{2}(B \cdot \omega) u^{\mu}-\frac{C}{2} \epsilon^{\mu \nu \alpha \beta} u_{\nu} E_{\alpha} \omega_{\beta} .
$$

In the local rest frame where $u^{\mu}=(1, \mathbf{0})$, we have

$$
\begin{aligned}
& n_{\mathrm{CS}}=\frac{C}{2} \boldsymbol{B} \cdot \boldsymbol{\omega}, \\
& \boldsymbol{j}_{\mathrm{CS}}=\frac{C}{2} \boldsymbol{E} \times \boldsymbol{\omega} .
\end{aligned}
$$

Equations (30) and (31) agree with the results obtained by different approaches based on the Wigner function formalism [33] and the Landau level picture [13,34], respectively. We however emphasize that while the results 
of Refs. [13,33,34] were obtained for free Dirac fermions in the homogenous electromagnetic field, our derivation extends it to the case of interacting Dirac fermions in the generic inhomogeneous electromagnetic field. In particular, this derivation shows that the transport coefficients of Eqs. (28), (30)-(32) are completely fixed by the anomaly coefficient $C$ independently of interactions. This proves the conjecture of Ref. [13] that the coefficient of the magnetovorticity coupling in Eq. (31) is related to $C$. Equation (32) also indicates a new type of anomaly-related current, which does not require the presence of $\mu_{5}$ unlike the CME or other chiral transport phenomena. This is a dissipationless current which does not generate any entropy because $\boldsymbol{j} \cdot \boldsymbol{E}=0$.

Let us now consider the case with $|v| \ll 1$ in the inertial frame, for which $u^{\mu} \approx(1, v)$. In this case, we have the following contribution to the electric current in addition to Eq. $(32)^{5}$ :

$$
\Delta j_{\mathrm{CS}}=\frac{C}{2}(\boldsymbol{v} \cdot \boldsymbol{\omega}) \boldsymbol{B}+O\left(\boldsymbol{v}^{3}\right) .
$$

This is the HME. Again, our derivation shows that the transport coefficient of the HME is fixed by the anomaly coefficient $C$ and is exact independently of interactions.

We note that the expression of the HME is similar to that of the CME [5-8], $\boldsymbol{j}=C \mu_{5} \boldsymbol{B}$, where $\mu_{5} \equiv\left(\mu_{\mathrm{R}}-\mu_{\mathrm{L}}\right) / 2$ is the chiral chemical potential with $\mu_{\mathrm{R}, \mathrm{L}}$ being the chemical potentials for right- and left-handed fermions. We can see that the correspondence between the HME and CME is $\boldsymbol{v} \cdot \boldsymbol{\omega} \leftrightarrow \mu_{\mathrm{R}}-\mu_{\mathrm{L}}$. While it is natural to have such a correspondence since $\boldsymbol{v} \cdot \boldsymbol{\omega}$ has the same quantum number as $\mu_{\mathrm{R}}-\mu_{\mathrm{L}}$ [3], whether the prefactors are exactly the same is a priori nontrivial. We here show that this is indeed the case by revealing the relation of the HME to the chiral anomaly. Note also that the HME can be present even when $\mu_{5}=0$ unlike the CME.

\section{HELICAL PLASMA INSTABILITY}

One of the consequences of the HME is a new type of plasma instability, called the helical plasma instability [3]. Here, let us discuss the condition for the emergence of the HPI in details.

We consider a system that has a finite fluid helicity $n_{\mathrm{flu}}(\boldsymbol{x})=\boldsymbol{v} \cdot \boldsymbol{\omega}$ in some region. For simplicity, we assume that $|\boldsymbol{v}| \ll 1$ and the spatial variation of the fluid helicity is sufficiently small. In order to focus on the consequences of the HME, we set $\mu_{5}=0$.

\footnotetext{
${ }^{5}$ If $\partial_{t} \boldsymbol{v} \neq \mathbf{0}$, we would also have an analog of the anomalous Hall effect $\Delta \boldsymbol{j}_{\mathrm{CS}}=\frac{C}{4} \boldsymbol{E} \times\left(\boldsymbol{v} \times \partial_{t} \boldsymbol{v}\right)$. However, in the present derivation, this term is absent because of the constraint $\partial_{t} \boldsymbol{v}=\mathbf{0}$.
}

\section{A. Dissipationless fluids}

We first consider the ideal situation of relativistic fluids without any dissipation. Gauss's law and Ampère's law including the Chern-Simons current read

$$
\begin{gathered}
\boldsymbol{\nabla} \cdot \boldsymbol{E}=n_{\mathrm{CS}}+n_{\mathrm{back}}, \\
\boldsymbol{\nabla} \times \boldsymbol{B}=\boldsymbol{j}_{\mathrm{CS}}+\partial_{t} \boldsymbol{E},
\end{gathered}
$$

where $n_{\text {back }}$ is the background charge density and $\boldsymbol{j}_{\mathrm{CS}}$ is given by Eqs. (32) and (33). Below we assume the local charge neutrality $n_{\mathrm{CS}}+n_{\text {back }}=0$. Combining these equations with Faraday's law $\boldsymbol{\nabla} \times \boldsymbol{E}=-\partial_{t} \boldsymbol{B}$ and $\boldsymbol{\nabla} \cdot \boldsymbol{B}=0$, we obtain

$$
\left(\partial_{t}^{2}-\nabla^{2}\right) \boldsymbol{B}=\sigma_{\mathrm{eff}} \boldsymbol{\nabla} \times \boldsymbol{B}+\frac{C}{2}(\boldsymbol{\omega} \cdot \boldsymbol{\nabla}) \boldsymbol{E},
$$

where we defined

$$
\sigma_{\text {eff }} \equiv C_{\mathrm{HME}} n_{\mathrm{flu}}=\frac{n_{\mathrm{flu}}}{4 \pi^{2}}
$$

and assumed that spatial variation of $\omega$ is sufficiently small.

To see that Eq. (36) has an unstable mode, consider a specific configuration, $v_{x} \neq 0, v_{y}=v_{z} \approx 0$, and $\partial_{y} v^{z}=$ $-\partial_{z} v^{y}=\omega^{x} \neq 0$, such that $n_{\mathrm{flu}}=v^{x} \omega^{x} \neq 0$, in the region of interest. We then seek for a solution of the gauge field $\boldsymbol{A}$ in the helicity basis as

$$
\boldsymbol{A}_{ \pm}=\left(\boldsymbol{e}_{x} \pm \mathrm{i} \boldsymbol{e}_{y}\right) \mathrm{e}^{-\mathrm{i} \omega t+\mathrm{i} k z}
$$

where $\boldsymbol{e}_{x, y}$ are the unit vectors in the $x, y$ directions and the subscript \pm denotes the helicity $h= \pm 1$ states for $k>0$. Here, we chose the temporal gauge $A_{t}=0$ without loss of generality. It then follows that

$$
\boldsymbol{E}_{ \pm}=\mathrm{i} \omega \boldsymbol{A}_{ \pm}, \quad \boldsymbol{B}_{ \pm}= \pm k \boldsymbol{A}_{ \pm} .
$$

In this setup, the second term on the right-hand side of Eq. (36) vanishes, and the dispersion relations for $\boldsymbol{B}_{ \pm}$become

$$
\omega^{2}=k\left(k \mp \sigma_{\text {eff }}\right) .
$$

When $n_{\mathrm{flu}}>0$ and $n_{\mathrm{flu}}<0, \omega$ has the positive imaginary part in the region $0<k<\left|\sigma_{\text {eff }}\right|$ for the $h=1$ and $h=-1$ states, respectively. In particular, this imaginary part becomes maximal at $k_{\text {inst }}=\left|\sigma_{\text {eff }}\right| / 2$, for which the time evolution of the magnetic field is given by the exponentially growing behavior with the maximum exponent,

$$
B(t)=B(0) \mathrm{e}^{\left|\sigma_{\text {eff }}\right| t / 2} .
$$

Here $B(t)$ is the magnitude of the magnetic field at time $t$. Therefore, the typical length and timescales of the HPI in this case are 


$$
\ell_{\text {inst }}=t_{\text {inst }}=\frac{8 \pi^{2}}{\left|n_{\text {flu }}\right|}
$$

Similarly to the CPI, the magnetic field generated as a consequence of this HPI has finite helicity (positive helicity for $n_{\text {flu }}>0$ and negative helicity for $n_{\text {flu }}<0$ ), which is characterized by the magnetic helicity

$$
h_{\mathrm{mag}} \equiv \int \mathrm{d}^{3} \boldsymbol{x} \boldsymbol{A} \cdot \boldsymbol{B}
$$

In other words, the HPI is a dynamical process that converts the fluid helicity to the magnetic helicity and the fluid kinetic energy to the magnetic energy such that the total helicity and total energy are conserved [3].

\section{B. Dissipative fluids}

We then consider the case of dissipative fluids and take into account the contribution of the Ohmic current. In this case, the electric current is given by

$$
\boldsymbol{j}=\sigma(\boldsymbol{E}+\boldsymbol{v} \times \boldsymbol{B})+\frac{C}{2}[(\boldsymbol{v} \cdot \boldsymbol{\omega}) \boldsymbol{B}+\boldsymbol{E} \times \boldsymbol{\omega}],
$$

where $\sigma$ is the electrical conductivity. Combining it with Ampère's law $\boldsymbol{\nabla} \times \boldsymbol{B}=\boldsymbol{j}$ (where the displacement current $\partial_{t} \boldsymbol{E}$ can be ignored; see footnote 6), we have

$$
\boldsymbol{E}=-\boldsymbol{v} \times \boldsymbol{B}+\eta \boldsymbol{\nabla} \times \boldsymbol{B}-\frac{C \eta}{2}[(\boldsymbol{v} \cdot \boldsymbol{\omega}) \boldsymbol{B}+\boldsymbol{E} \times \boldsymbol{\omega}],
$$

where $\eta \equiv 1 / \sigma$ is the resistivity. When $\eta|\omega| \ll 1$, this equation can be solved in terms of $\boldsymbol{E}$ as

$$
\begin{aligned}
\boldsymbol{E}= & -\boldsymbol{v} \times \boldsymbol{B}+\eta \boldsymbol{\nabla} \times \boldsymbol{B}-\frac{C \eta}{2}(\boldsymbol{\omega} \cdot \boldsymbol{B}) \boldsymbol{v} \\
& +\frac{C \eta^{2}}{2}[\boldsymbol{\nabla}(\boldsymbol{\omega} \cdot \boldsymbol{B})-(\boldsymbol{\omega} \cdot \boldsymbol{\nabla}) \boldsymbol{B}],
\end{aligned}
$$

where we used $(\boldsymbol{v} \times \boldsymbol{B}) \times \boldsymbol{\omega}-(\boldsymbol{v} \cdot \boldsymbol{\omega}) \boldsymbol{B}=-(\boldsymbol{\omega} \cdot \boldsymbol{B}) \boldsymbol{v}$. Note here that the term involving the fluid helicity disappears due to the cancellation between the contributions from the currents $(\boldsymbol{v} \cdot \boldsymbol{\omega}) \boldsymbol{B}$ and $\boldsymbol{E} \times \boldsymbol{\omega}$ with the same transport coefficients. The terms with the coefficient $C \eta^{2} / 2$ in Eq. (46) are higher order in derivatives and will be ignored below. By inserting Eq. (46) into Faraday's law, we obtain

$$
\partial_{t} \boldsymbol{B}=\boldsymbol{\nabla} \times(\boldsymbol{v} \times \boldsymbol{B})+\eta \boldsymbol{\nabla}^{2} \boldsymbol{B}+\frac{C \eta}{2} \boldsymbol{\nabla} \times[(\boldsymbol{\omega} \cdot \boldsymbol{B}) \boldsymbol{v}] .
$$

In this case, since there is no parity-violating term involving fluid helicity, it does not exhibit the HPI unlike Eq. (36).

\section{Condition for the helical plasma instability}

The origin of this qualitative difference of dissipative fluids from dissipationless ones in Sec. V B is that the electric field is a dependent variable of the magnetic field as given by Eq. (46) and it can be integrated out. In terms of the notion of generalized global symmetries [35], this stems from the fact that the electric one-form symmetry is explicitly broken in medium and the electric field is no longer a low-energy degree of freedom in the usual formulation of magnetohydrodynamics $[36,37]$. What distinguishes the two scenarios is whether the typical time scale of the HPI, $t_{\text {inst }}$ in Eq. (42), is sufficiently small compared with the inverse of the gap of the electric field, given by $\eta^{6}$ : the HPI becomes relevant when $t_{\text {inst }} \ll \eta$, or equivalently, $n_{\mathrm{flu}} \gg \sigma$.

In the case of weakly coupled QED plasma at finite temperature $T$, the mean free path $\ell_{\mathrm{mfp}}$ and conductivity $\sigma$ are given parametrically by $[39,40]$

$$
\ell_{\mathrm{mfp}} \sim \frac{1}{e^{4} T}, \quad \sigma \sim e^{2} T^{2} \ell_{\mathrm{mfp}} \sim \frac{T}{e^{2}},
$$

except for logarithmic correction, where we restored the coupling constant $e$. On the other hand, $n_{\mathrm{flu}}$ is estimated as

$$
n_{\mathrm{flu}} \sim \frac{v^{2}}{L},
$$

where $L$ is the typical length scale for the variation of the hydrodynamic variables and $v$ is the typical magnitude of the fluid velocity. In the hydrodynamic regime where $L \gg \ell_{\text {mfp }}$, we have $n_{\text {flu }} \ll \sigma$ at the weak coupling $e \ll 1$, and hence, there is no HPI.

Note however that the discussion so far is limited to the weakly coupled plasma with massless Dirac fermions in the hydrodynamic regime. The following are two examples where this discussion does not apply and the HPI can appear:

(i) Strongly coupled plasma: in the case of quark-gluon plasmas (QGP), the parametric dependence of $\sigma$ is changed to [40]

$$
\ell_{\mathrm{mfp}} \sim \frac{1}{g^{4} T}, \quad \sigma \sim e^{2} T^{2} \ell_{\mathrm{mfp}} \sim \frac{e^{2} T}{g^{4}},
$$

where $g$ is the QCD coupling constant. Although this estimate is obtained by the weak-coupling analysis and is applicable to $g \ll 1$ strictly speaking, if we

\footnotetext{
${ }^{6}$ The fact that the electric field has a gap $\sigma$ can be seen as follows [38]: by substituting the Ohmic law $\boldsymbol{j}=\boldsymbol{\sigma} \boldsymbol{E}$ into the continuity equation $\partial_{t} n+\boldsymbol{\nabla} \cdot \boldsymbol{j}=0$ and by using the Gauss law $\boldsymbol{\nabla} \cdot \boldsymbol{E}=n$, one obtains $\partial_{t} n+\sigma n=0$. Therefore, $n \propto \mathrm{e}^{-\sigma t}$, showing the presence of the gap $\sigma$. We also have $\left|\partial_{t} \boldsymbol{E}\right|=\eta\left|\partial_{t}\right| \ll|\boldsymbol{j}|$ for the timescale $t \gg \eta$, which justifies the assumption that the displacement current in the Ampère's law is negligible.
} 
extrapolate this formula to $g \gtrsim 1$, there is a regime where $n_{\text {flu }} \gg \sigma$ is satisfied. To see this, we rewrite the mean free path as $\ell_{\mathrm{mfp}} \sim e^{2} /\left(g^{8} \sigma\right)$ from Eq. (50). The hydrodynamic condition $L \gg \ell_{\text {mfp }}$ then yields $n_{\text {flu }} \ll v^{2} g^{8} \sigma / e^{2}$. To satisfy the condition for HPI simultaneously, it is required that $g^{4} \gg e / v$, which could be satisfied in strongly coupled QCD, but can never be satisfied in QED, for which $g$ is replaced by $e$. Therefore, the HPI can emerge in the strongly coupled QGP with a finite fluid helicity.

(ii) Nonrelativistic corrections: the fact that both of transport coefficients of the currents $(\boldsymbol{v} \cdot \boldsymbol{\omega}) \boldsymbol{B}$ and $\boldsymbol{E} \times \boldsymbol{\omega}$ are given by the same factor related to the chiral anomaly, $C / 2$, is specific to the case for massless Dirac fermions and is not generically applicable to the case for massive Dirac fermions and nonrelativistic particles. Since these two currents are related to each other via Lorentz transformation as can be understood from Eq. (30), the cancellation of the two should not be complete, e.g., for a nonrelativistic particle. In this case, the remaining term proportional to $(\boldsymbol{v} \cdot \boldsymbol{\omega}) \boldsymbol{B}$ leads to the HPI in a way similar to Eq. (36). Such a case is relevant to core-collapse supernovae (see below).

\section{DISCUSSIONS AND OUTLOOK}

In this paper, we have shown that the coefficients of the HME and the magnetovorticity coupling are completely fixed by the anomaly coefficient. Although the HME looks similar to the CME, the prominent feature of the HME is that it exists even when $\mu_{5}=0$. This is phenomenologically important because while $\mu_{5}$ is attenuated by a small but finite fermion mass, which could then suppress the CME, e.g., in core-collapse supernovae [41] and the electroweak plasma in the early Universe $[42,43]$, the fluid helicity is not.

In the context of core-collapse supernovae, fluid helicity can be generated through the CVE of the neutrino in local thermal equilibrium, $\boldsymbol{j}=-\left(\frac{\mu_{\nu}^{2}}{4 \pi^{2}}+\frac{T^{2}}{12}\right) \boldsymbol{\omega}$ with $\mu_{\nu}$ the neutrino chemical potential $[32,44]$, as pointed out in Ref. [3]. ${ }^{7}$

\footnotetext{
${ }^{7}$ We note that the generation of fluid helicity is not necessarily limited to the situation where neutrinos are in local thermal equilibrium. In fact, it has been recently shown using the chiral kinetic theory for neutrinos coupled to hydrodynamics for matter (dubbed the chiral radiation transfer theory) that the neutrinomatter collision can generate fluid helicity of matter even when neutrinos are away from equilibrium [45].
}

Then, the resulting fluid helicity induces the HME for relativistic electrons and nonrelativistic protons, and the former transport coefficient is given by Eq. (2) (with a possible small mass correction). While the HME for electrons presumably does not lead to HPI, the HME for protons may lead to HPI as argued above. This could be a potential mechanism for the inverse energy cascade in corecollapse supernovae [46]. It would also be interesting to study the possible helical instability beyond the hydrodynamic regime of neutrinos.

How efficiently the fluid helicity is generated in these systems is a nonlinear problem and should be investigated numerically by the helical magnetohydrodynamics incorporating the helical effects [3]. To this end, one first needs to extend the helical magnetohydrodynamics to the second order by including the HME and other possible effects. It is especially important to explore the possible anomalyrelated corrections to the energy-momentum tensor at the second order.

From the theoretical viewpoints, it would be interesting to study how the topological quantization of the transport coefficient of the HME and that of the magnetovorticity coupling can be understood in terms of the Berry curvature in a way similar to that of the CME [47-49]. It would also be interesting to derive these second-order helical transport phenomena from the underlying quantum field theory based on the Wigner function formalism, such as Refs. [50-52], beyond the homogeneous electromagnetic fields considered in Ref. [33].

Finally, we note that we assumed massless Dirac fermions in this paper. When Dirac fermions dynamically acquire a finite mass via interactions, the 't Hooft anomaly matching condition requires that there must be other gapless modes in the system that are responsible for the chiral anomaly. In this case, one expects that the HME is carried by these gapless modes just as the CME and CVE are carried by Nambu-Goldstone modes [53,54]. Accordingly, there must be an analog of the WessZumino-Witten term $[55,56]$ for the HME. This question is deferred to future work.

\section{ACKNOWLEDGMENTS}

We thank Koichi Hattori for discussions and Taro Kimura for comments. This work was supported by the Keio Institute of Pure and Applied Sciences (KiPAS) project at Keio University and JSPS KAKENHI Grants No. 19K03852 and No. 20K14470. 
[1] D. E. Kharzeev, J. Liao, S. A. Voloshin, and G. Wang, Prog. Part. Nucl. Phys. 88, 1 (2016).

[2] M. Joyce and M. E. Shaposhnikov, Phys. Rev. Lett. 79, 1193 (1997).

[3] N. Yamamoto, Phys. Rev. D 93, 065017 (2016).

[4] P. Hosur and X. Qi, C.R. Phys. 14, 857 (2013).

[5] A. Vilenkin, Phys. Rev. D 22, 3080 (1980).

[6] H. B. Nielsen and M. Ninomiya, Phys. Lett. 130B, 389 (1983).

[7] A. Y. Alekseev, V. V. Cheianov, and J. Frohlich, Phys. Rev. Lett. 81, 3503 (1998).

[8] K. Fukushima, D. E. Kharzeev, and H. J. Warringa, Phys. Rev. D 78, 074033 (2008).

[9] H. K. Moffatt, J. Fluid Mech. 35, 117 (1969).

[10] A. Yoshizawa, Hydrodynamic and Magnetohydrodynamic Turbulent Flows: Modelling and Statistical Theory (Kluwer Academic, Dordrecht, 1998).

[11] D. E. Kharzeev, Y. Kikuchi, R. Meyer, and Y. Tanizaki, Phys. Rev. B 98, 014305 (2018).

[12] V. E. Ambrus and M. N. Chernodub, arXiv:1912.11034.

[13] K. Hattori and Y. Yin, Phys. Rev. Lett. 117, 152002 (2016).

[14] A. Shitade and T. Kimura, Phys. Rev. B 90, 134510 (2014).

[15] Z. V. Khaidukov and M. A. Zubkov, JETP Lett. 108, 670 (2018).

[16] I. L. Shapiro, Phys. Rep. 357, 113 (2002).

[17] J. M. Luttinger, Phys. Rev. 135, A1505 (1964).

[18] S. Imaki and Z. Qiu, Phys. Rev. D 102, 016001 (2020).

[19] Y. Ferreiros and K. Landsteiner, arXiv:2011.10535.

[20] J. L. Mañes, M. Valle, and M. Á. Vázquez-Mozo, arXiv: 2012.08449.

[21] Y. Akamatsu and N. Yamamoto, Phys. Rev. Lett. 111, 052002 (2013).

[22] K. Landsteiner, Acta Phys. Pol. B 47, 2617 (2016).

[23] G. Basar, D. E. Kharzeev, and H. U. Yee, Phys. Rev. B 89, 035142 (2014).

[24] K. Landsteiner, Phys. Rev. B 89, 075124 (2014).

[25] K. Y. Yang, Y. M. Lu, and Y. Ran, Phys. Rev. B 84, 075129 (2011).

[26] A. G. Grushin, Phys. Rev. D 86, 045001 (2012).

[27] A. A. Zyuzin and A. A. Burkov, Phys. Rev. B 86, 115133 (2012).

[28] P. Goswami and S. Tewari, Phys. Rev. B 88, 245107 (2013).

[29] E. V. Gorbar, V. A. Miransky, I. A. Shovkovy, and P. O. Sukhachov, Phys. Rev. Lett. 118, 127601 (2017).

[30] E. V. Gorbar, V. A. Miransky, I. A. Shovkovy, and P. O. Sukhachov, Phys. Rev. B 97, 121105 (2018).
[31] N. Yamamoto, Phys. Rev. D 92, 085011 (2015).

[32] K. Landsteiner, E. Megias, and F. Pena-Benitez, Phys. Rev. Lett. 107, 021601 (2011).

[33] S. Z. Yang, J. H. Gao, Z. T. Liang, and Q. Wang, Phys. Rev. D 102, 116024 (2020).

[34] S. Lin and L. Yang, arXiv:2103.11577.

[35] D. Gaiotto, A. Kapustin, N. Seiberg, and B. Willett, J. High Energy Phys. 02 (2015) 172.

[36] S. Grozdanov, D. M. Hofman, and N. Iqbal, Phys. Rev. D 95, 096003 (2017).

[37] P. Glorioso and D. T. Son, arXiv:1811.04879.

[38] P. A. Davidson, An Introduction to Magnetohydrodynamics (Cambridge University Press, Cambridge, England, 2001).

[39] G. Baym, H. Monien, C. J. Pethick, and D. G. Ravenhall, Phys. Rev. Lett. 64, 1867 (1990).

[40] P. B. Arnold, G. D. Moore, and L. G. Yaffe, J. High Energy Phys. 11 (2000) 001.

[41] D. Grabowska, D. B. Kaplan, and S. Reddy, Phys. Rev. D 91, 085035 (2015).

[42] A. Boyarsky, V. Cheianov, O. Ruchayskiy, and O. Sobol, Phys. Rev. Lett. 126, 021801 (2021).

[43] A. Boyarsky, V. Cheianov, O. Ruchayskiy, and O. Sobol, Phys. Rev. D 103, 013003 (2021).

[44] A. Vilenkin, Phys. Rev. D 20, 1807 (1979).

[45] N. Yamamoto and D. L. Yang, Astrophys. J. 895, 56 (2020).

[46] Y. Masada, K. Kotake, T. Takiwaki, and N. Yamamoto, Phys. Rev. D 98, 083018 (2018).

[47] D. T. Son and N. Yamamoto, Phys. Rev. Lett. 109, 181602 (2012).

[48] M. A. Stephanov and Y. Yin, Phys. Rev. Lett. 109, 162001 (2012).

[49] D. T. Son and N. Yamamoto, Phys. Rev. D 87, 085016 (2013).

[50] Y. Hidaka, S. Pu, and D. L. Yang, Phys. Rev. D 95, 091901 (2017).

[51] Y. Hidaka, S. Pu, and D. L. Yang, Phys. Rev. D 97, 016004 (2018).

[52] T. Hayata, Y. Hidaka, and K. Mameda, J. High Energy Phys. 05 (2021) 023.

[53] K. Fukushima and K. Mameda, Phys. Rev. D 86, 071501 (2012).

[54] X. G. Huang, K. Nishimura, and N. Yamamoto, J. High Energy Phys. 02 (2018) 069.

[55] J. Wess and B. Zumino, Phys. Lett. 37B, 95 (1971).

[56] E. Witten, Nucl. Phys. B223, 422 (1983). 